\title{
Radially Localized Measurements of Superthermal Electrons Using Oblique Electron Cyclotron Emission
}

\author{
S. Preische, P. C. Efthimion, S. M. Kaye
}

It is shown that radial localization of optically thin Electron Cyclotron Emission from superthermal electrons can be imposed by observation of emission upshifted fromthe thermal cyclotron resonance in the horizontal midplane of a tokamak. Anew and unique diagnostic has been proposed and operated to make radially localized measurements of superthermal electrons during Lower Hybrid Current Drive on the PBX-M tokamak. The superthermal electron density profile as well as moments of the electron energy distribution as a function of radius are measured during Lower Hybrid Current Drive. The time evolution of these measurements after the Lower Hybrid power is turned off are given and the observed behavior reflects the collisional isotropization of the energy distribution and radial diffusion of the spatial profile.

\section{DISCLAIMER}

This report was prepared as an account of work sponsored by an agency of the United States Government. Neither the United States Government nor any agency thereof, nor any of their employees, makes any warranty, express or implied, or assumes any legal liability or responsibility for the accuracy, completeness, or usefulness of any information, apparatus, product, or process disclosed, or represents that its use would not infringe privately owned rights. Reference herein to any specific commercial product, process, or service by trade name, trademark, manufacturer, or otherwise does not necessarily constitute or imply its endorsement, recommendation, or favoring by the United States Government or any agency thereof. The views and opinions of authors expressed herein do not necessarily state or reflect those of the United States Government or any agency thereof. 


\section{DISCLAIMER}

Portions of this document may be illegible in electronic image products. Images are produced from the best available original document. 


\title{
Radially Localized Measurements of Superthermal Electrons Using Oblique Electron Cyclotron Emission
}

\author{
S. Preische*, P. C. Efthimion, S. M. Kaye \\ Princeton University \\ Plasma Physics Laboratory \\ P.O. Box 451, Princeton, N.J. 08543-0451
}

\begin{abstract}
It is shown that radial localization of optically thin Electron Cyclotron Emission from superthermal electrons can be imposed by observation of emission upshifted from the thermal cyclotron resonance in the horizontal midplane of a tokamak. A new and unique diagnostic has been proposed and operated to make radially localized measurements of superthermal electrons during Lower Hybrid Current Drive on the PBX-M tokamak. The superthermal electron density profile as well as moments of the electron energy distribution as a function of radius are measured during Lower Hybrid Current Drive. The time evolution of these measurements after the Lower Hybrid power is turned off are given and the observed behavior reflects the collisional isotropization of the energy distribution and radial diffusion of the spatial profile.
\end{abstract}

PACS: $52.70 . \mathrm{Gw}, 52.25 . \mathrm{Sw}, 52.50 . \mathrm{Gj}, 52.55 . \mathrm{Fa}$

*Present address: Association Euratom-CEA sur la Fusion, Centre d'Etudes de Cadarache, 13108 St. Paul-lez-Durance, France 


\section{Introduction}

The route to advanced tokamak operations which is presently envisioned relies on control of the current density profile across the tokamak minor radius by non-inductive means, as well as control of the pressure profile. ${ }^{1,2}$ Since macroscopic control of the current profile depends on the detailed behavior of the electron distribution function, and especially a superthermal population produced by non-inductive current drive, measurements of the radial behavior of the current-carrying part of this distribution, as well as its energy content are of interest. In this work a new diagnostic method is developed, both theoretically and experimentally on the PBX-M tokamak, to make such spatially localized measurements of the fast electron distribution. It is found that radially localized emission from superthermal electrons may be obtained by an oblique view of Electron Cyclotron Emission (ECE) upshifted from a low harmonic of the local electron cyclotron frequency in the horizontal midplane of the tokamak. Experiments have been previously performed using the electron cyclotron resonance to measure properties of the superthermal distribution, ${ }^{3-7}$ however they have been unable to make spatially localized measurements. Spatial localization of upshifted emission is imposed in this work by the region of parameter space (frequency, magnetic field, and observation angle) in which the electron cyclotron resonance can be fulfilled. The sensitivity of the ECE resonance condition to changes in this parameter space allows probing of the distribution function in energy and physical space, and thus radially localized measurements of energy parameters of the distribution as well as measurement of the superthermal density profile.

In Section II the technique of radial localization by Oblique ECE is presented and a model for oblique emission is given. The diagnostic arrangement on PBX-M is discussed in Section III. Section IV presents measurements made with the Oblique Electron Cyclotron Emission diagnostic and their analysis to obtain radially localized moments of the superthermal electron distribution. Conclusions and further applications of this technique are presented in in Section V.

\section{Oblique Electron Cyclotron Emission}

While a non-relativistic thermal plasma radiates ECE at harmonics of the local cyclotron frequency, an energetic population of electrons present in a plasma will radiate energy in a range of frequencies around these harmonics according to the relativistic electron 
cyclotron resonance condition. The resonance condition is given by

$$
\gamma-n_{\|} u_{\|}=\frac{m \Omega_{c e}}{\omega}
$$

where

$$
n_{\|}^{2}+\left(\frac{m \Omega_{c e}}{\omega}\right)^{2}-1 \geq 0
$$

$\gamma$ is the relativistic factor, the normalized momentum $u_{\|}=p_{\|} / m c$ so that $\gamma^{2}=1+u^{2}, m$ is the harmonic number, $\Omega_{c e}$ is the local electron cyclotron frequency given by the electron rest mass, and $\omega$ is the resonant frequency. The condition given by Eqn. 2 is the necessary condition for real solutions to the dispersion relation; that is, there is no electron cyclotron emission (or absorption) in a region of parameter space (frequency, magnetic field, and observation angle) where Eqn. 2 is not fulfilled.

Many other experiments which use electron cyclotron emission/absorption to measure properties of superthermal electrons use a perpendicular view $\left(n_{\|}=0\right){ }^{3,4,7}$ In this case, Eqn. 2 states that only downshifted emission may be observed, making it difficult to set spatial limits on the region of emission. Transmission experiments are performed at a finite angle to the magnetic field, ${ }^{5,6}$ however these experiments also use an interaction at frequencies which are downshifted from the electron cyclotron frequency and thus cannot achieve radial localization. It is the use of upshifted emission which allows radial localization in the present case.

An outer radial limit on the spatial region in which observed ECE can originate can be set using Eqn. 2. When ECE is viewed in the mid-plane from the outside of the torus at an oblique angle to the magnetic field, emission which is upshifted from a low harmonic of the local electron cyclotron frequency can be observed. Equation 2 determines the outer radial limit of this upshifted emission, as illustrated by the upper right shaded region for second harmonic emission in Fig. 1 for typical PBX-M parameters. As this condition is dependent on the local electron cyclotron frequency, the spatial limit which it sets varies with frequency. Note that the region in which Eqn. 2 is not satisfied is not a region inaccessible to the wave; radiation can propagate through this region but cannot originate in it.

An inner radial limit on the spatial region of the observed emission may be achieved in two different ways. When emission is observed upshifted from an optically thick harmonic, such as second harmonic extraordinary-mode (X-mode), the thermal layer sets an inner radial limit on the spatial region of emission. Any emission originating inside the thermal layer $\left(R<R\left(\omega=m \Omega_{c e}\right)\right.$ ) would be reabsorbed by thermal electrons. Thus the observed emission is a combination of emission from thermal electrons and superthermal 
electrons. As the thermal emission would be known from any electron temperature profile measurement, the emission above the thermal level must be due to superthermal electrons in the radial region between the thermal layer and the location given by Eqn. 2 . The radial region thus determined by a view in the mid-plane would vary with frequency due to the variation in the radial location of the cyclotron frequency. Thus a scan in frequency provides a scan in radius of the.emission from superthermal electrons. This method relies on the thermal layer being optically thick.

A second means of setting an inner radial limit on emission can be used under tokamak operating conditions where $\omega_{p e} \sim \Omega_{c e}$. In such a case, second harmonic X-mode radiation from thermal electrons can be cut-off by the right-hand cutoff frequency. In this scenario, which is the typical operating regime of $\mathrm{PBX}-\mathrm{M}$ and other high- $\beta$ experiments, the right hand cut-off can be used to refract the emitted radiation. The turning point of a ray, determined by the right-hand cutoff

$$
\omega_{R H}=\frac{\Omega_{c e}}{2}\left(\sqrt{1+\frac{4 \omega_{p e}^{2}}{\Omega_{c e}^{2}\left(1-n_{\|}^{2}\right)}}+1\right),
$$

sets an inner radial limit on the spatial region from which emission at a particular frequency can originate. As the right-hand cut-off is dependent on the local electron density, different frequencies probe different spatial regions of the plasma. This is illustrated in Fig. 1. The asymmetric shape of the cutoff as a function of radius is due to the fact that the index of refraction changes from $n_{\|}=.54$ at the outer edge to $n_{\|}=.78$ on the inside for the chosen viewing angle. Emission at frequencies in the range $65-90 \mathrm{GHz}$ is shown to be radially localized under the given plasma conditions.

The viewing scenario in PBX-M for emission upshifted from the second harmonic of the electron cyclotron frequency is illustrated in Fig. 2 for a viewing angle of $57^{\circ}$ with respect to the toroidal direction. Shown in Fig. 2(b) is the emission in second harmonic as a function of minor radius at $80 \mathrm{GHz}$, assuming a radially constant distribution of superthermal electrons. Figure 2(b) illustrates the radial localization of the emission between the turning point of the ray, determined by Eqn. 3, and the outer limit imposed by Eqn. 2 . Emission at this viewing angle is also enhanced because of the forward peaking of emission from mildly relativistic electrons.

In addition to radial localization, changes in the parameters of the resonance condition (Eqn. 1) can be used to vary the region of energy space which is observed. Figure 3 illustrates possible resonance curves in energy space. It is seen that a change in the ratio $\frac{m \Omega_{c e}}{\omega}$ produces a significant change in the resonant perpendicular energies, while a change in the viewing angle $\left(n_{\|}\right)$, which necessitates a change in the ratio $\frac{m \Omega_{c s}}{\omega}$ to fulfill Eqn. 2, 


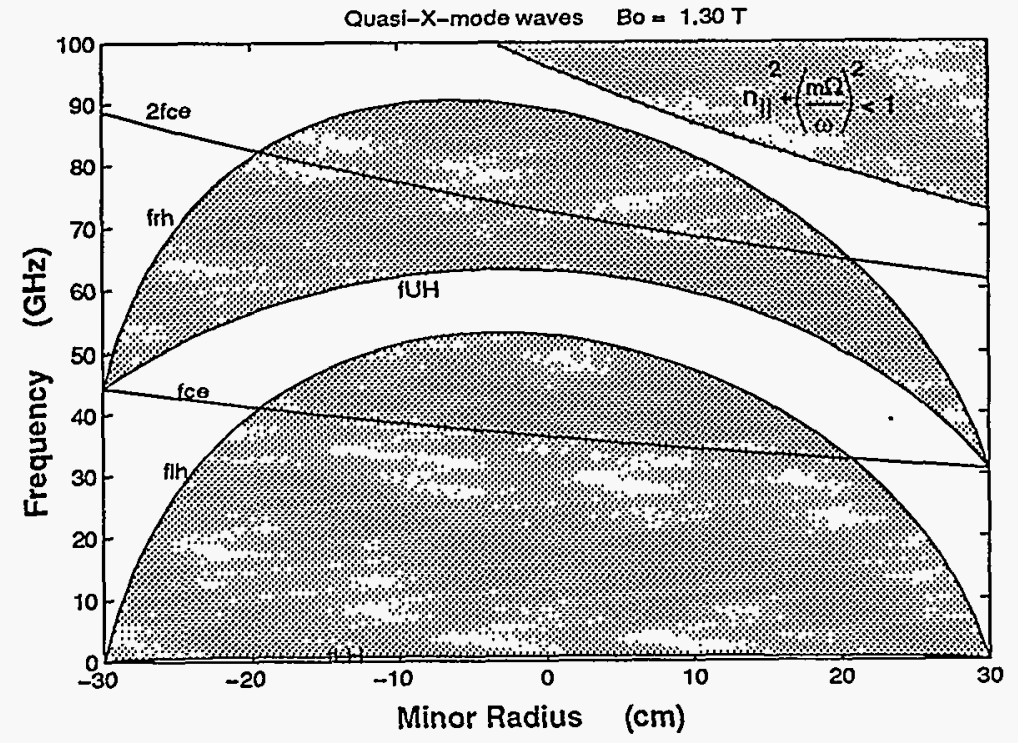

Figure 1: Accessibility diagram in the midplane for $P B X-M$ parameters under the plasma conditions $n_{e o}=3.3 \times 10^{13} \mathrm{~cm}^{-3}, B_{T o}=1.3 T$, and viewing angle $=57^{\circ}$ with respect to the toroidal direction. Emission cannot originate in the shaded regions. Upshifted second harmonic emission in the range $65-90 \mathrm{GHz}$ is spatially localized between the right-hand cutoff and the condition imposed by Eqn. 2 .

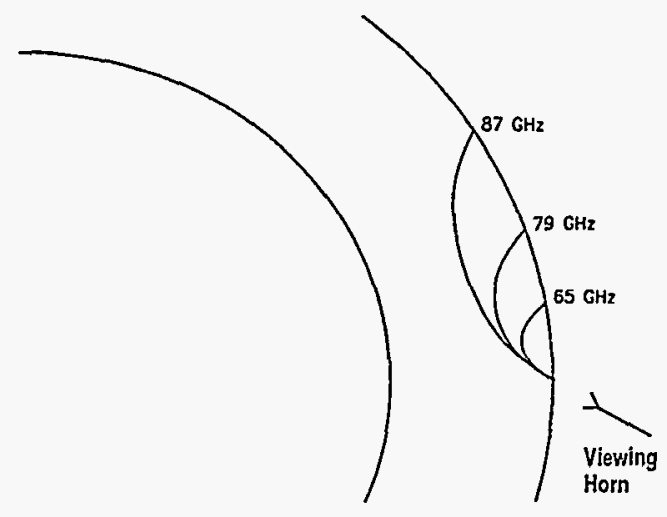

(a)

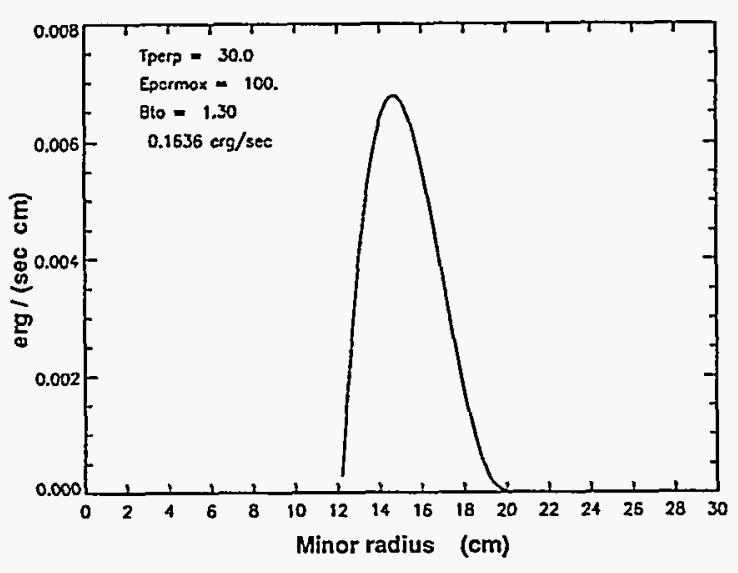

(b)

Figure 2: (a) Toroidal view of observation ray path, $n_{e o}=3.4 \times 10^{13} \mathrm{~cm}^{-3}, B_{T_{o}}=$ $1.3 T$, viewing angle $=57^{\circ}$ with respect to the toroidal direction. (b) Second harmonic emission along ray path at $80 \mathrm{GHz}$ given a fast electron energy distribution which is constant with radius with a density of $2 \times 10^{10} \mathrm{~cm}^{-3}$, a plateau to a high parallel energy cutoff of $100 \mathrm{keV}$ and a perpendicular temperature of $30 \mathrm{keV}$. 


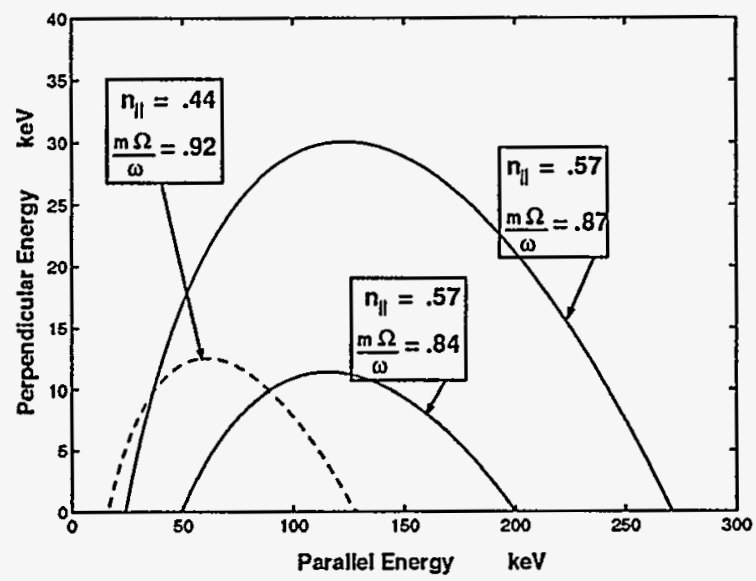

Figure 3: Solid lines illustrate the change in the resonance curve due to a change in the ratio $\frac{m \Omega_{c e}}{\omega}$ at a constant $n_{\|}$. The dotted line illustrates the change in the resonance curve due to a change in the viewing angle $\left(n_{\|}\right)$.

produces a change in the resonant parallel energies. An experiment in which both of these parameters are varied can most effectively determine the energy parameters of the distribution function.

The relation between the emission observed in such an experiment and the electron distribution function is shown in the following paragraphs. Once such a relation is established, moments of the distribution function can be determined from the observed emission. The total single pass emission observed along a ray path may be written ${ }^{8}$

$$
I(\omega)=\int_{\text {raypath }} d s \frac{1}{n_{r}^{2}(s)} \sum_{m=1}^{\infty} \beta_{m}(\omega, s) e^{-\tau_{m}(s)},
$$

where $n_{r}^{2}$ is the ray refractive index, $\beta_{m}$ is the local emissivity in the $m$ th harmonic, $\tau_{m}$ is the optical depth ( $\tau_{m}=\int^{s} \alpha_{m}(x) d x$, where $\alpha_{m}$ is the local absorption in the $m$ th harmonic), and the sum is over harmonics. Local properties of the ray are found by a ray tracing code. ${ }^{9}$ In cases where refractive effects are important, dielectric effects are not negligible, and a form of the emission and absorption coefficients must be used which includes such effects. The fully relativistic emission coefficient at a given spatial location has been derived from the linearized Klimontovich-Maxwell equations by Freund and $\mathrm{Wu} .{ }^{10}$ Using the resonance condition to reduce the problem to a one dimensional integral along the resonance curve, this emission coefficient may be written

$$
\beta_{m}(\omega, s)=\frac{n_{e} e^{2} \omega n_{r}^{2} \cos \xi \operatorname{Tr} \lambda Y_{m}^{2}}{\left|\frac{\partial}{\partial n^{2}} \Lambda_{r}\right| c n n_{\perp}^{2}|E|^{2}} \int_{u_{-}}^{u_{+}}\left|E_{x}+i E_{y}^{*}+\frac{n_{\perp} u_{\|}}{Y_{m}} E_{z}\right|^{2}\left[J_{m}^{2}(b) f\right]_{u_{\perp R}} d u_{\|},
$$

where $n_{e}$ is the electron density, $n$ is the total index of refraction, $Y_{m}=\frac{m \Omega_{c e}}{\omega}, E$ is the electric field of the wave, $\Lambda_{r}$ is the real part of the determinant of the dispersion tensor, 
$\operatorname{Tr} \lambda$ is the trace of the transpose of the cofactor matrix of the dispersion tensor, $f(\mathrm{u})$ is the momentum distribution function, $J_{m}$ is the $m$ th order Bessel function, $b=k_{\perp} \rho_{L}=$ $n_{\perp} u_{\perp} \omega / \Omega_{c e}, u_{ \pm}=\left[n_{\|} Y_{m} \pm \sqrt{n_{\|}^{2}-1+Y_{m}^{2}}\right] /\left(1-n_{\|}^{2}\right)$ are the resonant values for $u_{\perp}=0$, and $u_{\perp R}$ is defined by the resonance curve $u_{\perp R}^{2}=\left(n_{\|} u_{\|}+Y_{m}\right)^{2}-1-u_{\|}^{2}$. The integrand is only defined under the condition given by Eqn. 2 . Here the equation derived by Freund and $\mathrm{Wu}$ for an isotropic plasma is multiplied by the factor $d \Omega_{k} / d \Omega_{s}=n_{r}^{2} \cos \xi / n^{2},{ }^{8,11}$ where $d \Omega_{k}$ is the element of solid angle around the wave vector, $d \Omega_{s}$ is the element of solid angle around the group velocity vector, and $\xi$ is the angle between the group velocity and the wave vector $k$. This factor represents changes in the energy flux due to the anisotropy of the plasma. The local polarization of the wave is found from the local solution of the dispersion relation along the ray. It has also been assumed that the derivative of the Bessel function may be written: $J_{m}^{\prime}(b) \simeq \frac{m}{b} J_{m}(b)$, i.e. $\frac{m}{b} J_{m}(b) \gg J_{m+1}(b)$. Figure 3 gives an example of the resonance curves in energy space along which the integral is performed. The elements of the plasma dispersion tensor for a hot plasma can be calculated, assuming the background plasma is maxwellian, using the plasma dispersion function $Z\left(\zeta_{m}\right)$, where $\zeta_{m}=\left(\omega-m \Omega_{c e}\right) / k_{\|} v_{t h}$, following Stix. ${ }^{12}$

The absorption is found from the emissivity by the transformation ${ }^{8,13}$

$$
\frac{u_{\perp} f}{\gamma} \rightarrow-\frac{\delta \pi^{3} c^{2}}{n_{r}^{2} \omega^{2} m_{e} c^{2}} L_{m} f
$$

where

$$
\gamma L_{m} f=Y_{m} \frac{\partial f}{\partial u_{\perp}}+n_{\|} u_{\perp} \frac{\partial f}{\partial u_{\|}}
$$

it is given by

$$
\begin{aligned}
\alpha_{m}(\omega, s)= & -\frac{8 \pi^{3} n_{e} e^{2} \omega \cos \xi \operatorname{Tr} \lambda Y_{m}^{2}}{\left|\frac{\partial}{\partial n^{2}} \Lambda_{r}\right| \omega m_{e} c n n_{\perp}^{2}|E|^{2}} \times \\
& \int_{u_{-}}^{u_{+}}\left|E_{x}+i E_{y}^{*}+\frac{n_{\perp} u_{\|}}{Y_{m}} E_{z}\right|^{2}\left[\left(\frac{Y_{m}}{u_{\perp}} \frac{\partial f}{\partial u_{\perp}}+n_{\|} \frac{\partial f}{\partial u_{\|}}\right) J_{m}^{2}(b)\right]_{u_{\perp R}} d u_{\| \cdot}(6)
\end{aligned}
$$

Equations 5 and 6 are valid for all plasma regimes. In many cases the background plasma, which determines the dispersive properties of the medium, can be assumed to be cold, in which case the Altar-Appleton-Hartree dispersion relation ${ }^{12}$ can be used, and the emission and absorption coefficients can be reduced following Freund and Wu. ${ }^{10}$ The cold plasma approximation is valid when $k_{\perp} \rho_{L t h} \ll 1$ and $k_{\|\|} v_{t h} \ll \mid \omega-m \Omega_{c e}$, away from the thermal cyclotron layer. Note $k_{\perp} \rho_{L} \ll 1$ for the emitting electrons has not been assumed . The emitting electrons may be either thermal or a superthermal population. For a cold 
background plasma we find

$$
\beta_{m}(\omega, s)=\frac{n_{e} e^{2} \omega n_{r}^{2} \cos \xi}{c n} \frac{\left(\rho+\sin ^{2} \theta\right)}{2 \rho} \int_{u_{-}}^{u_{+}}\left[\left\{\gamma V_{ \pm} J_{m}(b)+u_{\perp} J_{m}^{\prime}(b)\right\}^{2} f\right]_{u_{\perp R}} d u_{\|}
$$

where

$$
\begin{aligned}
& V_{ \pm}=2 \frac{\omega}{\Omega_{c e}} \frac{\left(1-\frac{\omega_{p e}^{2}}{\omega^{2}}\right)\left(1-n_{\|} \beta_{\|}\right)-n_{\perp}^{2}}{n_{\perp}\left(\sin ^{2} \theta \mp \rho\right)} \\
& \rho^{2}=\sin ^{4} \theta+4 \frac{\omega^{2}}{\Omega_{c e}^{2}}\left(1-\frac{\omega_{p e}^{2}}{\omega^{2}}\right)^{2} \cos ^{2} \theta
\end{aligned}
$$

and

$$
\begin{aligned}
\alpha_{m}(\omega, s)= & -\frac{8 \pi^{3} n_{e} e^{2} \cos \xi}{m_{e} c \omega n} \frac{\left(\rho+\sin ^{2} \theta\right)}{2 \rho} \times \\
& \int_{u_{-}}^{u_{+}}\left[\left\{\gamma V_{ \pm} J_{m}(b)+u_{\perp} J_{m}^{\prime}(b)\right\}^{2}\left(\frac{Y_{m}}{u_{\perp}} \frac{\partial f}{\partial u_{\perp}}+n_{\|} \frac{\partial f}{\partial u_{\|}}\right)\right]_{u_{\perp R}} d u_{\| \cdot}
\end{aligned}
$$

The top sign refers to the O-mode and the bottom sign to the $\mathrm{X}$-mode.

$\mathrm{X}$ and $\mathrm{O}$-mode emission strictly refer to waves which propagate perpendicular to the magnetic field and are linearly polarized. For propagation at an oblique angle to the magnetic field the same terms will be used here to refer to waves which follow from the same branch of the dispersion relation. At an oblique angle, both $\mathrm{X}$ and $\mathrm{O}$-mode emission are elliptically polarized and thus both could be observed by a linearly polarized antenna. For the wave electric field vector defined with the $z$-direction along the magnetic field and the $x$-direction in the radial direction, a horizontal polarization is defined for oblique propagation: $E_{H}=E_{z} \sin \theta-E_{x} \cos \theta$, where $\theta$ is the angle of observation with respect to the toroidal field. The ellipticity of a wave propagating at $57^{\circ}$ with respect to the magnetic field is then found to be $\left|E_{y} / E_{H}\right|^{2}=1.67$ for the X-mode and $\left|E_{y} / E_{H}\right|^{2}=.6$ for the $\mathrm{O}$-mode. In general, the total power emitted into O-mode is lower than that into $\mathrm{X}$-mode. Calculations of observed power for a linearly polarized antenna show that for observation at a $57^{\circ}$ angle, with the observed electric field oriented in the vertical direction, $\left|E_{y}\right|^{2}$, the emission is predominantly in X-mode; less than $15 \%$ of the observed power is contributed by the O-mode.

In certain cases some harmonic overlap may occur. That is, third harmonic downshifted emission may also be observed from electron distribution functions which have a significant number of particles at high energies, particularly high perpendicular energies. For this reason, all calculations of emission will include the third harmonic contribution. 


\section{Experimental Setup}

The experiment described here was carried out on the PBX-M tokamak. As $\omega_{p e} \sim \Omega_{c e}$ under normal operating conditions on PBX-M, the method of refracting the observation rays from the right-hand cutoff was chosen to set the inner radial limit on the observed emission. This experiment was designed for a range of plasma parameters $n_{e o}=3.0-$ $3.7 \times 10^{13} \mathrm{~cm}^{-3}$ and $B_{T_{o}}=1.2-1.35$ Tesla. A viewing angle of $33^{\circ}$ with respect to the radial direction was chosen to optimize the radial localization of emission and the range of electron energies observed. Figure 1 illustrates the radial localization of electron cyclotron emission in the range $60-90 \mathrm{GHz}$ under these conditions. A superheterodyne radiometer ${ }^{14,15}$ was built to detect emission in this frequency range in either a swept or single frequency mode. The emission was received through a microwave antenna with a linearly polarized, corrugated scalar feed horn and a spot focused Gaussian lens in its 6 inch diameter aperture. The Gaussian beam was focused 35 inches in front of the aperture with a spot size of 1.1 inches at $75 \mathrm{GHz}$. The $-3 \mathrm{~dB}$ full beamwidth was found to be $2^{\circ}$ at the focus for $60 \mathrm{GHz}$, and will be smaller for higher frequencies. The $-10 \mathrm{~dB}$ point of the full beamwidth was found to be $6-7 \mathrm{~cm}$ along most of its path length through the plasma for $60 \mathrm{GHz}$.

For radial localization, it is important that the observed emission can be assumed to originate from a single pass through the plasma. In order to reduce wall reflections, microwave viewing dumps made of a Silicon Carbide $(\mathrm{SiC})$ based ceramic were installed along both the inner and outer walls of the vacuum chamber to provide coverage to at least the $-10 \mathrm{~dB}$ point of the viewing beam. Pyramids one inch in depth with an opening of $45^{\circ}$ were machined into the surface in order to increase microwave absorption by the viewing dumps. Reflection from these dumps was measured in the lab compared to the reflection from a metal plate. The dumps were found to reduce reflection by at least $-15 \mathrm{~dB}$, as com-

pared to the metal plate, for radiation incident at $45^{\circ}$ and up to $-30 \mathrm{~dB}$ for perpendicularly incident radiation. The arrangement of the viewing dumps and the microwave antenna in PBX-M is shown in Fig. 4.

To test the effectiveness of the viewing dumps, thermal emission from ohmic discharges with high and low density were compared. For the high density case, the plasma density and magnetic field were the same as those shown in Fig. 1. Second harmonic thermal emission above $65 \mathrm{GHz}$ is cut off under these conditions, and it is expected that no first pass emission should be observed. Perpendicular thermal emission at these frequencies, however, is not cut off. This difference is due to the dependence of the cutoff frequency on $n_{\|}$, shown in Eqn. 3. If the dumps are not working or the antenna is not viewing them, 


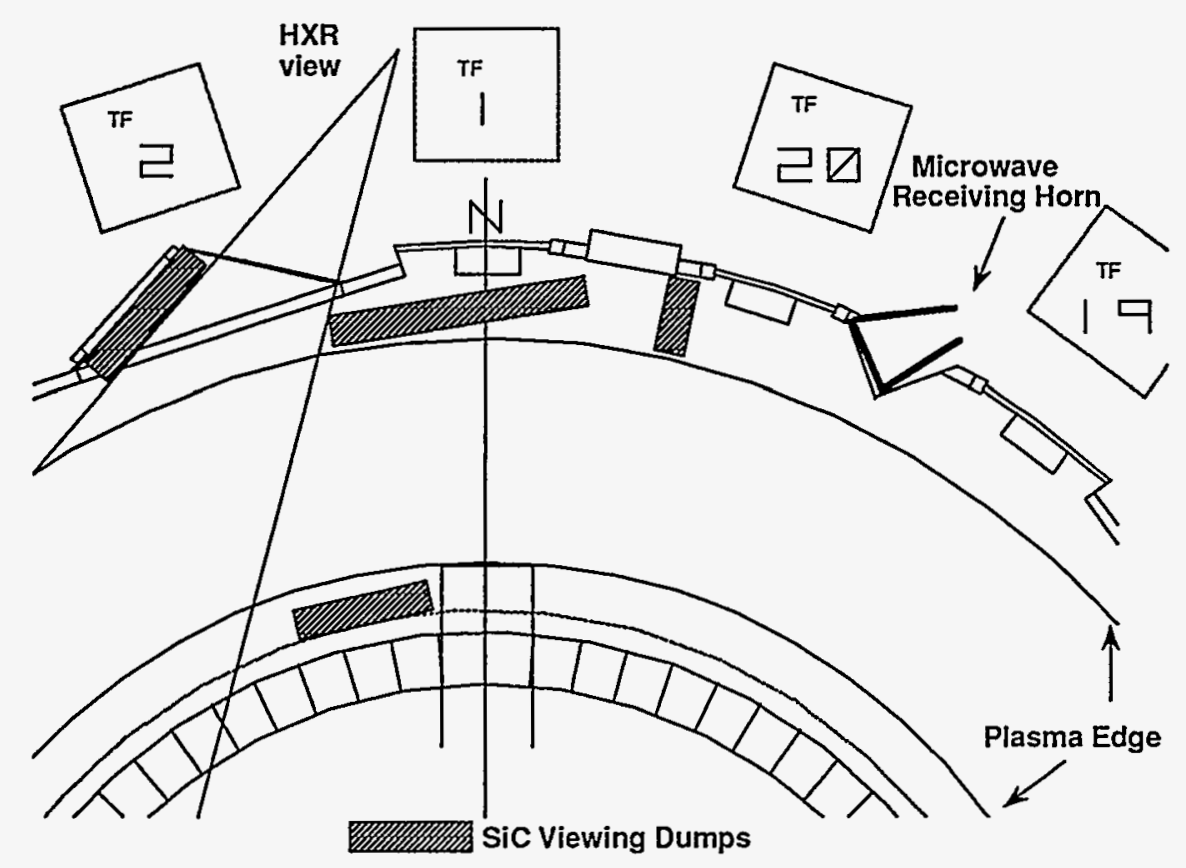

Figure 4: Toroidal view of PBX-M showing the arrangement of the microwave antenna and the $\mathrm{SiC}$ viewing dumps for the Oblique Electron Cyclotron Emission diagnostic.

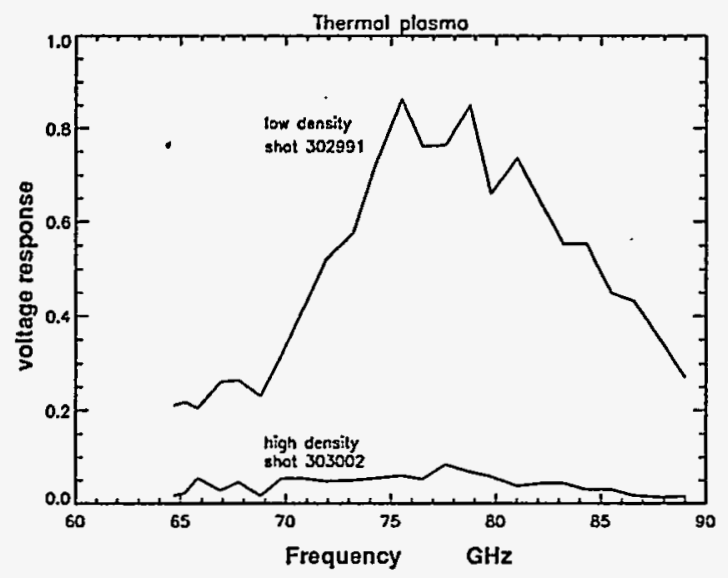

Figure 5: Lower line shows measured emission from a high density ohmic plasma for which the thermal emission is cut off, $n_{e o}=3 . \times 10^{13} \mathrm{~cm}^{-3}, T_{e o}=700 \mathrm{eV}, B_{T_{o}}=$ 1.3T. The upper line shows the thermal emission from a low density plasma where the thermal layer can be observed, $n_{e o}=1 . \times 10^{13} \mathrm{~cm}^{-3}, T_{e o}=900 \mathrm{eV}, B_{T_{o}}=1.3 \mathrm{~T}$. The low level of emission in the high density case demonstrates that the viewing dumps are effective.

this perpendicular emission could easily be reflected back into the ray path. The lower line in Fig. 5 shows the measured emission under these high density conditions. This case was 

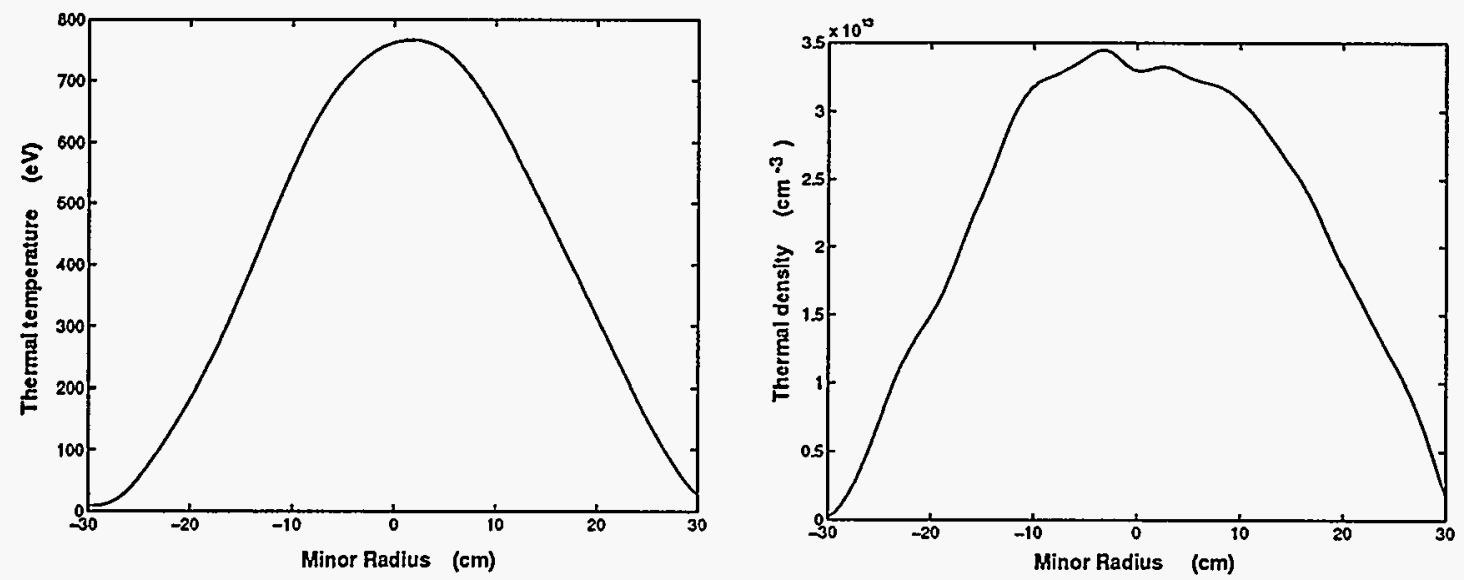

Figure 6: Typical temperature and density profiles for the shots used in this analysis. PBX-M shots 312021 - 312060.

compared with a low density ohmic discharge in which the thermal layer is not cutoff at the set observation angle. Emission under these conditions is shown in the upper line in Fig. 5. Since very little emission is observed under the high density conditions (approximately $10 \%$ of that observed under low density conditions), it is concluded that the ray path is bending as expected and the antenna is viewing the outer dumps under high density conditions.

\section{Measurements \& Analysis}

A series of measurements taken by the Oblique Electron Cyclotron Emission diagnostic will be analyzed here to determine moments of the electron distribution function as a function of radius and time. The parameters of the background plasma during these shots were $n_{e o}=3.2-3.6 \times 10^{13} \mathrm{~cm}^{-3}, T_{e o}=725-775 \mathrm{eV}, B_{T_{o}}=1.2-1.35 \mathrm{~T}$, and a plasma current of $180 \mathrm{kAmps}$. Typical background temperature and density profiles are shown in Fig. 6. Lower Hybrid power was launched into these plasmas with a phasing of $-82^{\circ}$ and power of $200 \mathrm{~kW}$. This phasing corresponds to a peak in the launched $n_{\|}$spectrum at 1.8. The current driven by Lower Hybrid waves provides only a small fraction of the total plasma current under these conditions ( $20-50 \mathrm{kAmps}$ ), and is well peaked off axis, as verified by Hard X-Ray measurements.

Small changes in both the thermal electron density and the toroidal magnetic field were used to control the location of the emission in both physical space and energy space. Measurements taken both at different magnetic fields and at different frequencies change the ratio $\frac{m \Omega_{c e}}{\omega}$ in the resonance condition (Eqn. 1). In this way the energy of the resonant elec- 
trons can be varied to improve the measurement's resolution of the electron distribution's energy parameters.

Because the observed emission is dependent on both the energy distribution and the density of the superthermal electrons, the energy distribution and its radial variation must be found before the radial profile of the superthermal density can be determined. As the emission is linearly related to the density, this density dependence can be easily eliminated by using ratios of measurements taken at the same radial location. Thus, we start by finding the energy distribution.

The emission can be expressed using, for example, Eqns. 4 and 7. Assuming all terms due to local plasma parameters are known, this emission depends only on the electron distribution function. The ratio of emission measured at two frequencies originating in the same spatial region then depends only on the energy distribution function. Thus in principle, the measurement of two such ratios gives a unique solution for an energy distribution which is modeled in terms of two parameters. However, measurements made close to each other in frequency space lie close to each other in solution space. Given the limited number of measurements able to be made during this experiment (measurements at one angle only), and given experimental uncertainties in the measurements, recovering the unique solution becomes impossible in this case.

A solution for the energy distribution is found with the help of Bayes' Theorem which takes into account the known uncertainties in the measurements and allows the calculation of the most probable solution and its uncertainty. Bayes' Theorem states

$$
P(\Theta \mid \mathrm{D})=\frac{P(\mathrm{D} \mid \Theta) P(\Theta)}{P(\mathrm{D})},
$$

where $P(D \mid \Theta)$ is the conditional probability distribution of the observed data $\mathbf{D}$ given a model with parameters $\Theta$ and some uncertainty in the measurement, $P(\Theta)$ represents the prior distribution of $\Theta$ (i.e. what is known about $\Theta$ before any measurements are taken), and $P(\mathbf{D})$ is the total probability of the measurement $\mathbf{D}$ :

$$
P(\mathrm{D})=\int P(\mathrm{D} \mid \Theta) P(\Theta) d \Theta .
$$

If $\mathrm{H}(\Theta)$ represents the model for the observed data given parameters $\Theta$, and the errors in the measurements are assumed to be distributed according to a Gaussian distribution, then

$$
P(\mathrm{D} \mid \Theta)=\frac{1}{(2 \pi)^{k / 2}|\mathrm{~V}|^{1 / 2}} e^{-\frac{1}{2}(\mathrm{D}-\mathrm{H}(\Theta))^{T} \mathrm{~V}^{-1}(\mathrm{D}-\mathrm{H}(\Theta))},
$$

with $\mathrm{V}$ the covariance matrix of $\mathrm{H}$, and both $\Theta$ and $\mathrm{D}$ have length $k$. In practice, $\mathrm{V}$ is taken to be the covariance matrix of the measurements $\mathrm{D}$ to include experimental uncer- 
tainties. In the present case, $\mathrm{H}$ is the model for the emission, given for example by Eqn. 4, and is dependent on the parameters $\Theta$ of the energy distribution.

The information available from the measurements made here is not enough to determine an arbitrary distribution function; the determination of a distribution of arbitrary form would require many more measurements. Thus an appropriate model for the distribution under Lower Hybrid Current Drive is chosen and the moments of that model are determined. The model energy distribution function which was chosen is defined in terms of a parallel distribution and a perpendicular temperature, where the perpendicular distribution is assumed to be Maxwellian:

$$
f\left(u_{\|}, u_{\perp}, r\right)=n_{s t h}(r) \frac{\mu\left(u_{\|}, r\right)}{2 \pi} \frac{f_{\|}\left(u_{\|}, r\right)}{\int f_{\|}\left(u_{\|}, r\right) d u_{\|}} e^{-\mu\left(u_{\|}, r\right) u_{\perp}^{2} / 2},
$$

where

and

$$
\mu\left(u_{\|}, r\right)=\frac{m_{e} c^{2}}{T_{\perp}\left(u_{\|}, r\right)}
$$

$$
f_{\|}\left(u_{\|}, r\right)=\int_{0}^{\infty} f\left(u_{\|}, u_{\perp}, r\right) 2 \pi u_{\perp} d u_{\perp} .
$$

For a model of the parallel distribution during Lower Hybrid Current Drive, a flat plateau distribution is chosen with a small percentage of the superthermal electron density "past the end" of the plateau. The parameters of the model are $E_{\| \max }$, which defines the end of the flat plateau region, and $T_{\perp o}$, which defines the perpendicular temperature in the plateau region. In the plateau region

$$
f_{\|}\left(E_{\|}, r\right)=1, \quad T_{\perp}\left(E_{\|}, r\right)=T_{\perp o} \quad 0<E_{\|}<E_{\| \max }(r) .
$$

For parallel energies higher than $E_{\| \max }$, the distribution is chosen to be

$$
\begin{gathered}
f_{\|}\left(E_{\|}, r\right) \propto e^{-.045\left(E_{\|}-E_{\| \max }(r)\right)} \quad E_{\|}>E_{\| \max }(r) \\
T_{\perp}\left(E_{\|}, r\right)=T_{\perp 0}\left(1.6-\frac{\left(E_{\|}-E_{\| \max }(r)-70\right)^{2}}{8166}\right) \quad E_{\|}>E_{\| \max }(r) .
\end{gathered}
$$

This shape of the distribution function given in Eqns. 14 and 15 was chosen to be similar to the distributions found by Fokker-Planck code predictions ${ }^{16}$ under similar PBX-M conditions. It was found that the solution in energy space for the parameters of the observed distribution function was insensitive to the exact form of the distribution for parallel energies higher than $E_{\| \text {max }}$.

Also included in the model of the emission is a Gaussian spread in $n_{\|}$in order to approximate a Gaussian beam in the plasma. This distribution is given by

$$
\beta_{m}(\omega, s)=\frac{1}{\sqrt{2 \pi} \sigma} \int_{-\infty}^{\infty} e^{-\left(n_{\|}-n_{\| o}\right)^{2} / 2 \sigma^{2}} \beta_{m}\left(n_{\|}, \omega, s\right) d n_{\|}
$$


where $n_{\|_{0}}$ is that along the central ray and the standard deviation is $\sigma=.01$. It was.found that such a spread in $n_{\|}$does not change the solution for the energy distribution function, however it does slightly broaden the error bars.

Bayes' Theorem allows the inclusion of any prior knowledge about the solution space through the distribution $P(\Theta)$ in Eqn. 9. In this case, the prior knowledge is taken to be that the maximum energy in the plateau of the energy distribution cannot exceed the energy of the electrons on which the launched $n_{\|}$would damp. Thus the distribution $P(\Theta)$ is zero for negative energies, constant from zero to the maximum energy allowed in the plateau, in this case $100 \mathrm{keV}$, and drops off exponentially above the energy defined by the launched $n_{\|}$in the same manner as the parallel distribution function defined by Eqn. 14. This prior distribution is not used in radial regions assumed to be outside the Lower Hybrid damping region.

For the limited number of frequencies and angles measured, three radial regions of emission are defined: $r_{\text {out }}: r>22 \mathrm{~cm}, r_{\text {mid }}: 16 \mathrm{~cm}<r<22 \mathrm{~cm}$, and $r_{\text {in }}: r<16 \mathrm{~cm}$ (where the total minor radius $a=30 \mathrm{~cm}$ ). When only second harmonic emission is observed, emission in frequencies lower than $69 \mathrm{GHz}$ originates in the outer radial region, frequencies $76-79 \mathrm{GHz}$ originate in the middle region, and frequencies above $83 \mathrm{GHz}$ originate in the inner radial region. Assuming that the energy distribution is constant in each region, energy parameters of the distribution are determined in each region. Measurements of the emission at three frequencies which are $1.5 \mathrm{GHz}$ apart are used in each region. The ratios of the emission measured at different frequencies are taken to eliminate the density dependence. The model $\mathrm{H}$ for this ratio of emission measurements is given by using Eqn. 7 in Eqn. 4 , where $\beta_{m}$ is dependent on the parameters $\Theta=\left[E_{\| \max }, T_{\perp_{0}}\right]$ and $\tau_{m} \ll 1$. The two ratios thus measured (D) and the model for these ratios are used with Bayes' Theorem to find the moments of the energy distribution in each radial region. Where possible, the two ratios are taken at different magnetic fields to increase the energy resolution. An example of the solution for the two moments of the energy distribution, $E_{\| \text {max }}$ and $T_{\perp o}$, in the middle radial region is shown in Fig. 7. The solution is the maximum of the distribution $P(\Theta \mid D)$. Error bars on this solution can be defined at the $1 \sigma$ level, given by the .67 confidence level. The full solutions for the moments of the energy distribution as a function of radius and as a function of time before and after the Lower Hybrid power was turned off, are shown in Table 1.

The most notable characteristic of the solution for the energy distribution and its time evolution is in the inner radial region, which covers the inside half of the plasma. The perpendicular temperature in this region increases with time after the Lower Hybrid power is turned off. This increase can be attributed to the relaxation of the distribution function 


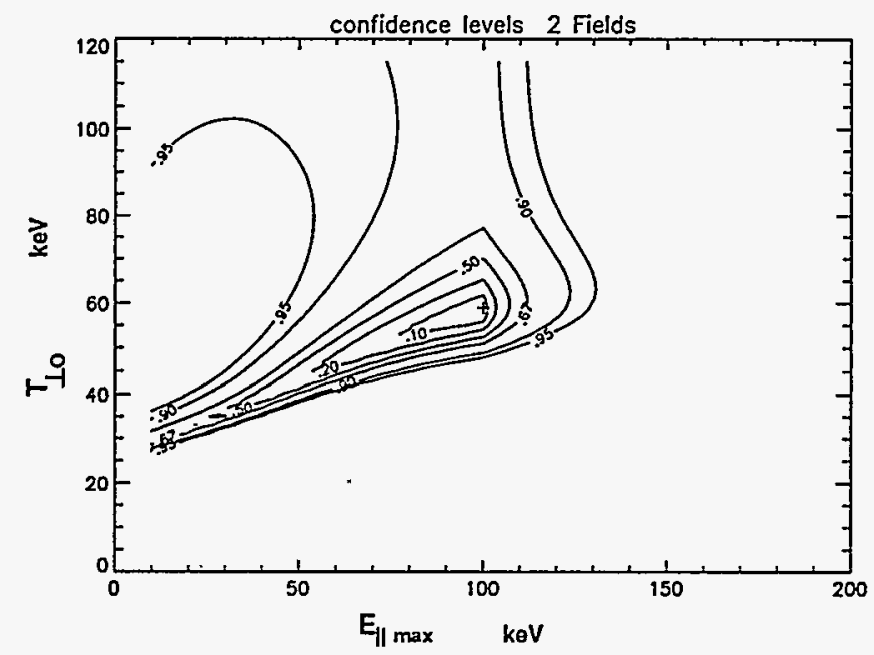

Figure 7: Confidence levels given by $P(\Theta \mid \mathrm{D})$ found from Eqn. 9 (where $\Theta=$ $\left.\left[E_{\| \max }, T_{\perp_{0}}\right]\right)$ for middle radial region while the Lower Hybrid power is on, time = 417 in Table 1. The most probable solution is the maximum of the distribution, marked by + .

\begin{tabular}{|c||c|c|c||c|c|c|}
\hline \multicolumn{1}{|c||}{} & \multicolumn{3}{c||}{$T_{\text {1o }}$} & \multicolumn{3}{c|}{$E_{\| \text {max }}$} \\
\hline \hline time & $r_{\text {in }}$ & $r_{\text {mid }}$ & $r_{\text {out }}$ & $r_{\text {in }}$ & $r_{\text {mid }}$ & $r_{\text {out }}$ \\
\hline 417 & 49 & 59 & 6 & 98 & 100 & 122 \\
423 & 49 & 53 & 7 & 98 & 100 & 136 \\
429 & 67 & 55 & 5 & 100 & 100 & 164 \\
435 & 67 & 55 & 5 & 100 & 100 & 164 \\
\hline
\end{tabular}

Table 1: Solution for moments of the energy distribution as a function of radius and time given by the maximum of $P(\Theta \mid D)$ in Eqn. 9. The Lower Hybrid power is turned off at 420 . Times are given in ms and energies in $\mathrm{keV}$.

due to pitch angle scattering. In the absence of an electric field, the collisional evolution of the average pitch angle is expected to occur on a time scale faster than the collisional slowing down of the velocity by a factor of $\left(1+Z_{\text {eff }}\right) \cdot{ }^{17}$ Although an electric field is present in this case, a similar effect can be expected. An increase in the perpendicular temperature of the distribution function, on a similar time scale, has also been observed on Tore Supra after the Lower Hybrid power is turned off. ${ }^{18}$ The energy distribution measured here is also in agreement with Hard X-Ray measurements of the energy distribution on PBX-M which have determined the parallel energy of the distribution to be between 100 $150 \mathrm{keV}$ under very similar conditions. ${ }^{19}$

After the energy distribution is found in each region, the absolute density profile is found from the absolute intensity of the measured emission. Six emission intensities ( 3 fre- 


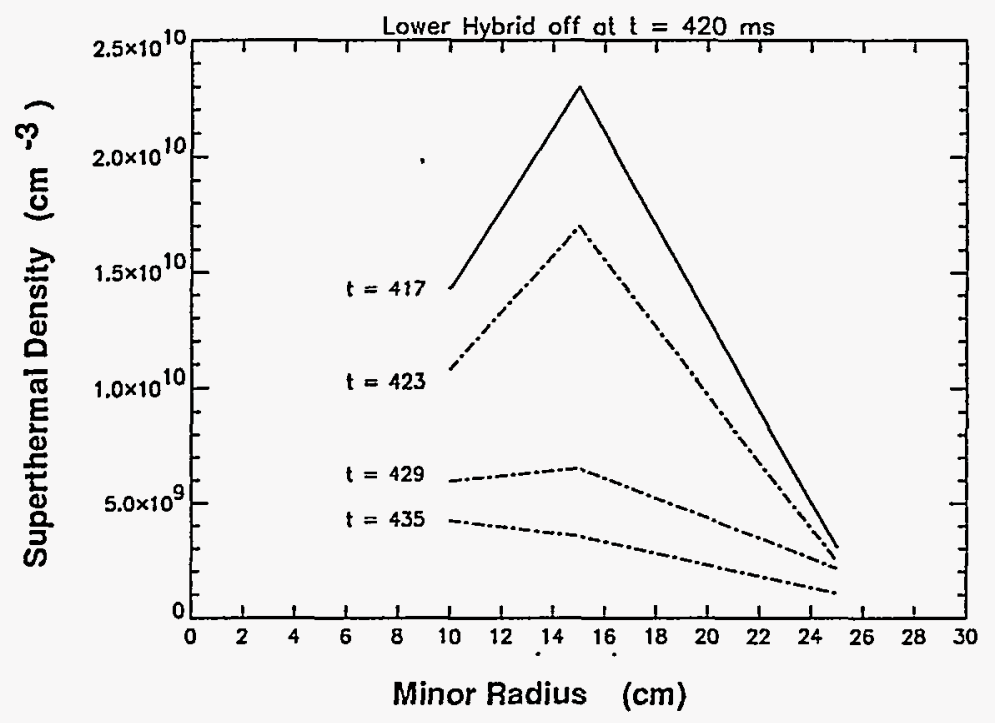

Figure 8: Radial profile of the superthermal density measured by the Oblique ECE system. The dotted lines show the time evolution of the density profile after the Lower Hybrid power is turned off at $420 \mathrm{~ms}$.

quencies $\times 2$ magnetic fields) were taken in each radial region and all of these measurements were used to find the density profile. The slope of the density profile was fitted in the middle and inner regions, using the absolute measurement found in the outer region. The location of the change in slope of the density profile is taken to be at $r=15 \mathrm{~cm}$ because this is the radial location at which the peak in the Hard X-Ray emissivity occurs. This is the only piece of information which is obtained from the Hard X-Ray data in this analysis. The density profiles obtained in this manner are shown in Fig. 8. It is seen that the time evolution of the measured profile exhibits radial diffusion of the fast electrons, as the peak of the distribution moves from off axis during Lower Hybrid Current Drive to on axis $15 \mathrm{~ms}$ after the Lower Hybrid power is turned off. A comparison of the measured density profile with the Hard X-Ray emissivity profile is shown in Fig. 9. The inversion of the $2 \mathrm{D}$ Hard X-Ray camera image is performed in the midplane only and removes the geometry effects of the view. ${ }^{20}$ The superthermal density profile measured by the Oblique ECE system is in very good agreement with the Hard X-Ray emissivity profile. 


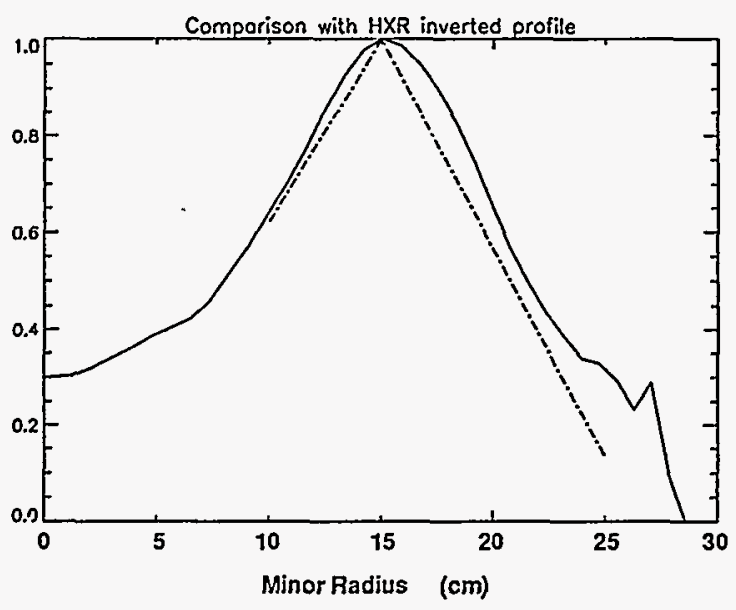

Figure 9: Solid line shows the normalized inverted Hard X-ray emissivity profile on the outer midplane while the Lower Hybrid power is on. Dotted line shows the normalized density profile measured by Oblique ECE at the same time.

\section{Conclusions \& Extensions}

A new and unique diagnostic has been proposed; developed, and operated on the PBX$M$ tokamak and has demonstrated that an oblique view of Electron Cyclotron Emission upshifted from a harmonic of the thermal cyclotron resonance in the horizontal midplane imposes radial localization on the observed emission. Radially localized emission from superthermal electrons has been obtained for the first time, allowing radially localized moments of the superthermal electron distribution to be made. This includes the first absolute measurement of the superthermal density profile. The observed time evolution of these measurements shows the collisional isotropization of the energy distribution and radial diffusion of the spatial profile after the Lower Hybrid power is turned off.

Further application of this new technique would be greatly enhanced by the ability to make measurements at more than one observation angle. In the discussion surrounding Fig. 3 , it has been demonstrated that changes in the observation angle would significantly improve the resolution in parallel energy of the measurement. Two methods of setting the inner radial limit on the emission have been outlined so that this technique can be applied to tokamak scenarios in which the right-hand cutoff is not near the second harmonic resonance as well as to cases where the second harmonic resonance is cutoff, as on PBX-M. This new measurement can thus have application to a wide range of experiments planned to achieve current profile control in advanced tokamak operating regimes and will contribute to a better understanding of the dynamics of superthermal electrons. 


\section{Acknowledgments}

This work was supported by DoE contract No. DE-AC02-76-CHO-3073.

We would like to thank $\mathrm{C}$. Forest for initial discussion about the geometry used here, A.

Kritz and G. Smith for the use of the TORCH ray-tracing code, and G. Giruzzi for Fokker-

Planck analysis of PBX-M scenarios. 


\section{References}

${ }^{1}$ Kessel, C. et. al. Physical Review Letters 72 (1994) 1212.

${ }^{2}$ Ferron, J. R. et. al. Physics of Fluids B 5 (1993) 2532.

${ }^{3}$ Luce, T.C. et. al. Review of Scientific Instruments 59 (1988) 1593.

${ }^{4}$ Kato, K. and I. Hutchinson, Physical Review Letters 56 (1986) 340.

${ }^{5}$ Kirkwood, R. et. al. Nuclear Fusion 30 (1990) 431.

${ }^{6}$ Skiff, F. et. al. Plasma Physics and Controlled Fusion, 36 (1994) 1371.

${ }^{7}$ Segui, J.L. and G. Giruzzi Plasma Physics and Controlled Fusion 36 (1994) 897.

${ }^{8}$ Bekefi, G. Radiation Processes in Plasmas, New York: John Wiley and Sons, 1966.

${ }^{9} \mathrm{Kritz}, \mathrm{A} . \mathrm{H}$. et. al. Heating in Toroidal Plasmas (Proceedings of 3rd Varenna-Grenoble Int. Symposium Grenoble, 1982) Vol. 2, CEC, Brussels (1982) 707.

${ }^{10}$ Freund, H. P. and C. S. Wu, Physics of Fluids 20 (1977) 963.

${ }^{11}$ Bornatici, M. et. al., Nuclear Fusion 23 (1983) 1153.

${ }^{12}$ Stix, T. H., Waves in Plasmas, New York: American Institute of Physics, 1992.

${ }^{13}$ Fidone, I. and G. Giruzzi, Nuclear Fusion 30 (1990) 803.

${ }^{14}$ Efthimion, P. C. et.al., Review of Scientific Instruments 50 (1979) 949.

${ }^{15}$ Taylor, G. et.al., Review of Scientific Instruments 55 (1984) 1739.

${ }^{16}$ Giruzzi, G. Plasma Physics and Controlled Fusion 35 (1993) A123.

${ }^{17}$ Fisch, N.J., Reviews of Modern Physics 59 (1987) 175.

${ }^{18}$ Giruzzi, G. et.al., Physical Review Letters 74 (1995) 550.

${ }^{19}$ von Goeler, S. et. al. Physics of Plasmas 2 (1995) 205.

${ }^{20}$ Bell, R.E. Review of Scientific Instruments 66 (1995). 